\title{
Mean Shift Gradient Vector Flow: A Robust External Force Field for 3D Active Surfaces
}

\author{
Margret Keuper, Hans Burkhardt, Olaf Ronneberger \\ Chair of Pattern Recognition and Image Processing and \\ Centre of Biological Signalling Studies (bioss) \\ University of Freiburg, Germany \\ keuper@informatik.uni-freiburg.de
}

\author{
Jan Padeken, Patrick Heun \\ Max-Planck Institute of Immunobiology \\ Freiburg, Germany and \\ Centre of Biological Signalling Studies (bioss) \\ University of Freiburg, Germany
}

\begin{abstract}
Gradient vector flow snakes are a very common method in bio-medical image segmentation. The use of gradient vector flow herein brings some major advantages like a large capture range and a good adaption of the snakes in concave regions. In some cases though, the application of gradient vector flow can also have undesired effects, e.g. if only parts of an image are strongly blurred, the remaining weak gradients will be smoothed away. Also, large gradients resulting from small but bright image structures usually have strong impact on the overall result. To tackle this problem, we present an improvement of the gradient vector flow, using the mean shift procedure and show its advantages on the segmentation of $3 D$ cell nuclei.
\end{abstract}

Keywords-diffusion; segmentation; deformable models; vector fields;

\section{INTRODUCTION}

Active surfaces are a widely used method in the field of bio-medical image segmentation tasks. Especially when dealing with 3D microscopic data, one has to handle several challenges as there are blurred object edges due to the point spread function (PSF) of the microscope as well as artifacts caused by inhomogeneities in the fluorescent stains. If the data comes from widefield microscopy, the PSF strongly blurs the recording and causes artifacts. To get acceptable segmentation results and to weaken the ill-posedness of the segmentation task, one often tries to include as much prior shape knowledge as possible into the model, e.g. [1], [5]. For the segmentation of DAPI-stained nuclei in a Drosophila cell line (so called S2 or Schneider cells) recorded with 3D widefield fluorescence microscopy, an active surface model has thus been adapted to the segmentation of sphere-like objects in [5]. In that paper, the force field has been adapted by projecting the gradients onto their radial components such that only the desired gradients have impact on the segmentation result. This indeed leads to better contours but still, artifacts can be observed. One of the major problems is that the resulting active surfaces seem to be elongated and subjected to PSF artifacts compared to what would be expected. See fig. 3 for an example of the data in two orthogonal views and the PSF of the used microscopy setting in xz-view. In [5], gradient vector flow (GVF) [2] was used to smooth the gradient field before adapting the active surfaces. This leads to some desired effects like a large capture range for the active surface model as well as smooth gradient fields. On the other hand, the weak but important gradients in upper and lower image regions are smoothed away by the classical GVF.

To diminish the artifacts caused by the dataset blurring, we are presenting a vector diffusion method that not only preserves strong image gradients but also preserves weak gradients if they are dense i.e. if all gradients in a neighborhood have similar direction and length. This method is the result of combining the basic GVF with the mean shift procedure [3], [4].

\section{GRAdient Vector Flow}

The currently best established method to smooth vector fields is the gradient vector flow (GVF)[2]. This diffusion method aims at providing a smooth vector field in regions, where no edges are given in the dataset, and in keeping the original gradient information in regions with strong edges. Thus, when the vector field is used as an external force field e.g. for active contours, the capture range is much larger than it would be, if the original gradient information were used. The three dimensional gradient vector flow field is the vector field $\mathbf{v}(\mathbf{x}): \mathbb{R}^{3} \rightarrow \mathbb{R}^{3}$ that minimizes the energy functional

$$
E(M)=\int_{\mathbb{R}^{3}} \mu\left(|\nabla \mathbf{v}|^{2}\right)+|\nabla M|^{2}|\mathbf{v}-\nabla M|^{2} \mathrm{~d} \mathbf{x},
$$

where $M$ is the edge map of the original dataset and the grad operator $\nabla$ is applied to each component of $\mathbf{v}$ separately. This energy minimization is solved by introducing a time variable $t$ and finding the steady-state solution of the partial differential equation (PDE) (compare [2]):

$$
\frac{\partial \mathbf{v}}{\partial t}=\mu \nabla^{2} \mathbf{v}-(\mathbf{v}-\nabla M)|\nabla M|^{2} .
$$

See fig. 1 for a toy example. The $2 \mathrm{D}$ toy data $1(\mathrm{a})(120 \times$ 120 pixels) is designed to simulate the blurred recordings of spherical objects with three undesired spots (spot 1-3) that should influence the overall force field as little as possible. The used edge image $M$ (fig. 1b) is the magnitude of the 
smoothed gradients of the toy data (object borders). The gradient field $\nabla M$ is shown in $1(\mathrm{c})$. Colors indicate the vector direction. In 1(d), the GVF field (300 iterations with $\mu=0.15$ ) of the toy data is displayed. Below the GVF in 1(d), we have plotted the profile of the original gradient field 1(c) (blue) and the GVF field (red) along the red line in 1(a).

\section{MEAN SHIFT FILTERING}

The mean shift procedure as presented in [3] aims at analyzing the density distribution of arbitrary feature spaces and can be used to detect density maxima or to perform filtering or clustering of feature points. The theoretical basis for the application of the procedure as an image filter has been presented in [4]. The prospect of applying the mean shift procedure on gradient vector fields is to analyze, which gradient vectors correspond to more important structures in the dataset and accordingly, to shift the gradient vectors towards their local density maxima. Thus, gradients corresponding to smaller, less important structures can be filtered out.

The mean shift algorithm is based on the kernel density estimation. Here, we briefly summarize the mean shift algorithm as described in [4].

\section{A. Kernel Density Estimation}

For $n$ independent data points $\mathbf{x}_{i}, i=1, \ldots, n$ in $d$ dimensional space $\mathbb{R}^{d}$, that are generated by an unknown probability distribution $f$, a kernel density estimate can be given by

$$
\hat{f}_{h, k}(\mathbf{x})=\frac{c_{k, d}}{n h^{d}} \sum_{i=1}^{n} k\left(\left\|\frac{\mathbf{x}-\mathbf{x}_{i}}{h}\right\|^{2}\right),
$$

where the function $k(x)$ is the radial profile of a $d$ dimensional kernel $K(\mathbf{x})$, that is a bounded function and integrates to one: $K(\mathbf{x})=c_{k, d} k\left(\|\mathbf{x}\|^{2}\right)$.

The function $k(x)$ with $k(x) \geq 0$ only needs to be defined for $x \geq 0$. The constant $c_{k, d}$ with $c_{k, d}>0$ ensures, that $K(\mathbf{x})$ integrates to one. $h$ is the kernel bandwidth parameter. For complexity reasons, we are using the Epanechnikov kernel (compare [4]), with the profile $k_{E}(x)=1-x$, for $0 \leq x \leq 1$ and 0 for $x>1$. $k_{E}$ is not differentiable for $x=1$, for $x \neq 1, k_{E}^{\prime}(x)$ is uniform.

The main step of the mean shift procedure is to determine local maxima in this density distribution of the feature space. Thus, zero positions of the gradient $\nabla f(\mathbf{x})=0$ are estimated by gradient ascent, which is done by the mean shift

$$
\mathbf{m}_{h, g}(\mathbf{y})=\hat{c} \frac{\nabla \hat{f}_{h, k}(\mathbf{y})}{\hat{f}_{h, g}(\mathbf{y})}=\frac{\sum_{i=1}^{n} \mathbf{x}_{i} g\left(\left\|\frac{\mathbf{y}-\mathbf{x}_{i}}{h}\right\|^{2}\right)}{\sum_{i=1}^{n} g\left(\left\|\frac{\mathbf{y}-\mathbf{x}_{i}}{h}\right\|^{2}\right)}-\mathbf{y},
$$

where $g(x)=-k^{\prime}(x)$ and $\hat{c}>0$ is constant. From (4), the gradient ascent iteration, shifting each vector towards the nearest local maximum, is derived as:

$$
\mathbf{y}_{j+1}=\mathbf{m}_{h, g}\left(\mathbf{y}_{j}\right)+\mathbf{y}_{j} .
$$

\section{B. Mean Shift on $3 D$ Vector Fields}

In [4], the authors show how the mean shift procedure can be employed as a discontinuity preserving image filter. This is done by interpreting the image as a two-dimensional lattice of $p$-dimensional vectors ( $p=1$ in the gray level case, $p=3$ for color images). The lattice then forms the spatial domain, the color information forms the range domain of a $d=p+2$ dimensional feature vector in the joint spatial-range domain. The mean shift is performed using a multivariate kernel

$$
K_{h_{s}, h_{r}}=\frac{C}{h_{s}^{2} h_{r}^{2}} k\left(\left\|\frac{\mathbf{x}^{s}}{h_{s}}\right\|^{2}\right) k\left(\left\|\frac{\mathbf{x}^{r}}{h_{r}}\right\|^{2}\right),
$$

defined as a product of two radially symmetric kernels with the common profile $k(x) . \mathbf{x}^{s}$ is the spatial part, $\mathbf{x}^{r}$ the range part of the feature vector. The Euclidean metric allows the use of a single bandwidth parameter in each domain, $h_{s}$ and $h_{r}$ respectively. These bandwidth parameters control the size of the kernel and thus determine the resolution of the density maximum detection. $C$ is the corresponding normalization constant.

An extension of the algorithm presented in [4] to the three dimensional space is straightforward. In the case of a three dimensional vector field $\nabla M\left(\mathbf{x}^{s}\right): \mathbb{R}^{3} \rightarrow \mathbb{R}^{3}$, where $M$ is a $3 \mathrm{D}$ scalar field, the mean shift is computed on the $\bar{d}=3+3$ dimensional feature vector, where the first three components contain the spatial information, the last three dimensions contain the corresponding vector information. As in the two dimensional case, the Epanechnikov kernel performs satisfactorily.

When applying this edge preserving mean shift filter to gradient vector fields, the result is quite similar to what we know from 2D color images. Depending on the chosen spatial and range bandwidth parameters, the discontinuities at the dataset gradients are preserved and the vectors converge to equal length and direction in the neighborhood defined by the spatial bandwidth parameter. See fig. 1(e) for the toy example $\left(h_{s}=6, h_{r}=0.4\right)$. Because of the round shape of the object, the gradient vectors converge to the mean direction in their neighborhood, i.e. all vectors are pointing towards the edges. The result is depending on the filter bandwidth. Vectors with $\left\|\mathbf{v}_{i}-\mathbf{v}_{j}\right\| \leq h_{r}$ converge to equal length if they are spatially close to one another. Maxima in gradient magnitude are not preserved. Find the profile along the red line below the mean shift filtered gradients 1(e).

\section{Mean Shift GVF}

With the GVF, an isotropic vector diffusion is performed. The regularization only depends on the vectors length disregarding their importance in their local neighborhood. 
In regions, where the gradients are generally weaker, the Laplace term dominates equation 1 and a strong smoothing is performed. In our example of the microscopic recordings of cell nuclei, this is not always desirable. First, we do not want to promote strong gradients, if they are originating from small structures. Second, in the strongly blurred upper and lower dataset regions, we want to keep the gradients pointing towards the cell nuclei even though they are weak. Generally, we want that large gradients originating from small structures are preserved but do not diffuse as strongly as in GVF, i.e. have less impact on the overall force field. On the other hand, if in a certain region there are many weak gradient vectors pointing into the same direction, these valuable gradients shall not be altered by larger neighboring gradients (as it would happen in GVF), but all gradients in the same neighborhood shall converge to their mean direction and length. This is why we are using the advantages of the mean shift filtering method and the GVF simultaneously, performing a minimization of the energy functional (1) and a maximization of the kernel density estimate (3) at the same time, which we call mean shift gradient vector flow (MSGVF). The new energy functional we are minimizing is given by

$$
E(M)=\int_{\mathbb{R}^{3}} \mu\left(|\nabla \overline{\mathbf{v}}|^{2}\right)+|\nabla M|^{2}|\overline{\mathbf{v}}-\nabla M|^{2} \mathrm{~d} \mathbf{x},
$$

where

$$
\overline{\mathbf{v}}=\mathbf{v}+\hat{c} \frac{\nabla \hat{f}_{h, k}(\mathbf{v})}{\hat{f}_{h, g}(\mathbf{v})}=\mathbf{v}+\mathbf{m}_{h, g}(\mathbf{v}),
$$

Thus, we are searching for a smooth vector field, where the original vector length is low, while at the same time searching for maxima of the kernel density, i.e. $\mathbf{m}_{h, G}(\mathbf{v})=$ 0 . By analogy to [2], this energy functional is minimized by the following Euler-Lagrange equation:

$$
\mu \nabla^{2}\left(\mathbf{v}+\mathbf{m}_{h, g}(\mathbf{v})\right)-\left(\mathbf{v}+\mathbf{m}_{h, g}(\mathbf{v})-\nabla M\right)|\nabla M|^{2}=0,
$$

with the resulting PDE

$$
\begin{aligned}
\frac{\partial \mathbf{v}}{\partial t}= & \mu \nabla^{2}\left(\mathbf{v}+\mathbf{m}_{h, g}(\mathbf{v})\right) \\
& -\left(\left(\mathbf{v}+\mathbf{m}_{h, g}(\mathbf{v})\right)-\nabla M\right)|\nabla M|^{2} .
\end{aligned}
$$

Unlike for GVF, the optimization cannot be done for each channel separately, because $\mathbf{m}_{h, g}(\mathbf{v})$ is depending of all channels. The result of the MSGVF can be seen in fig. 1(f), for 100 iterations with $\mu=0.15, h_{s}=6$ and $h_{r}=0.2$. As if applying GVF 1(d), the discontinuities are preserved and long gradients corresponding to object edges are unaltered. As opposed to GVF, gradients resulting from smaller objects have less impact on the overall result. This can be seen when looking at the streamline plot in fig. 2. Spot 1 does not change the vector direction at all. When looking at spot 3 , one can observe, that strong gradients are caused by this structure. These are preserved by both methods. Only the diffusion behavior is different. In the case of MSGVF, the

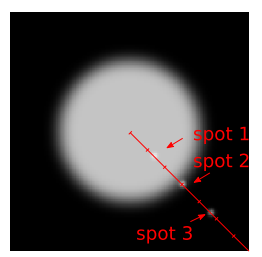

(a) toy data
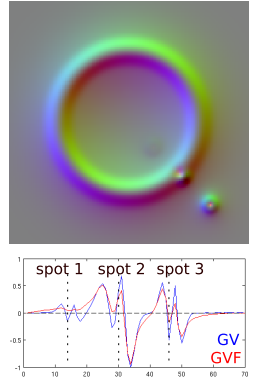

(d) GVF

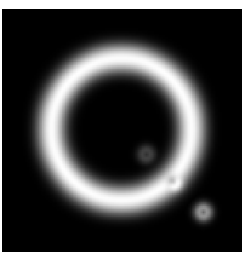

(b) edge map
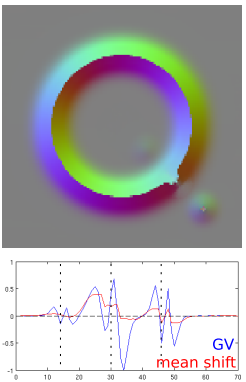

(e) Mean Shift filter

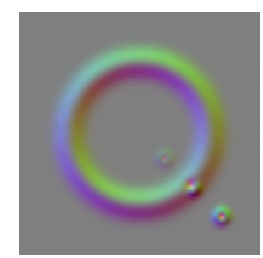

(c) gradient vectors
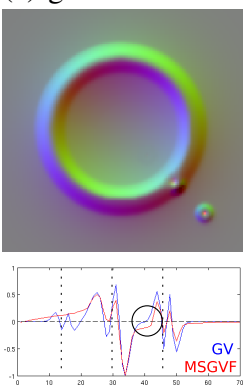

(f) MSGVF
Figure 1. The methods on toy data.

zero crossing (see profile), indicating the change of vector direction, is shifted towards spot 3 . The capture range for our main object is thus larger (see also the streamline plots). Even spot 2, lying directly on the boundary, has less impact on the vector field.

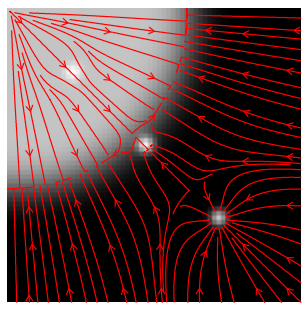

(a) Streamline plot of the GVF field in the ROI.

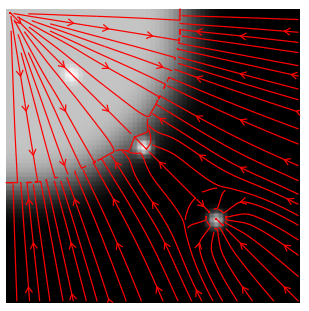

(b) Streamline plot of the MSGVF field in the ROI.
Figure 2. Especially when looking at the small spot outside the large object (spot 3), one can see that it has strong impact on the vector field, and this impact is significantly larger, when applying GVF (a), than when applying MSGVF (b). Thus, if we want to segment the main object, our initial surface must fit better with GVF than with MSGVF. Also, when applying MSGVF, spot 1 has no impact on the vector direction at all and even the capture range of spot 2 is less strong. So, both spots will not significantly alter the segmentation result.

\section{ApPlication AND Results}

We have applied the new method to generate force fields for the segmentation of Drosophila S2 cell nuclei, see fig. 3(a) for example. In [5], the last step of the force field generation is applying GVF to the vector field. We are showing that by replacing this step with the presented MSGVF, we can improve the segmentation and diminish the 
artifacts caused by the PSF. To allow for comparison, all further steps of the active surface procedure are the same as in [5]. For GVF as for MSGVF, we set $\mu=0.15$ in xydirection, which is the parameter advised in [2] for 3D GVF. In $\mathrm{z}$-direction, we scaled $\mu$ with the voxelsise, which is three times lower, than in xy-direction. The MSGVF parameters were set to $h_{s}=2$ and $h_{r}=0.2$. We performed 300 iterations for both GVF and MSGVF which was sufficient for convergence.

We applied our method to a dataset of 393 recordings of

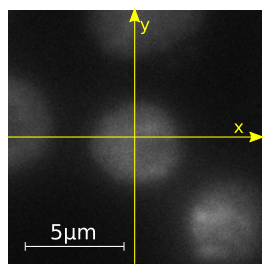

(a) Drosophila S2 nuclei in two orthogonal views.

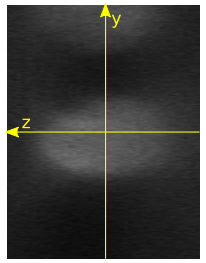

$2 \mu \mathrm{m}$

(b) Measured PSF in xz-view.
Figure 3. Microscopic data from widefield fluorescent microscopy.

Drosophila S2 cell nuclei. To measure the susceptibility of the segmentation surface to artifacts, we are locally approximating the surface by spheres and estimate their radii. At the best, most radii should be similar to the radius of the cells that is somewhere near $2 \mu \mathrm{m}$. The more artifacts we have in a surface, the more very small radii can be found. As we expect to find these artifacts in the lower dataset regions, we compute the measure of the lower $20 \%$ of the segmentation mask and compare it to the $20 \%$ in the center. The result is shown in fig. 4 . The results show

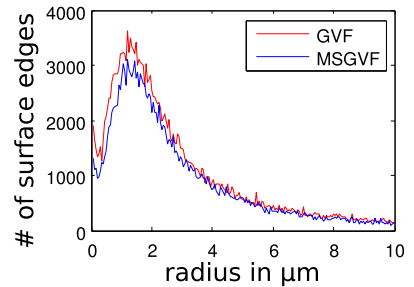

(a) Histogram of the radii in the center.

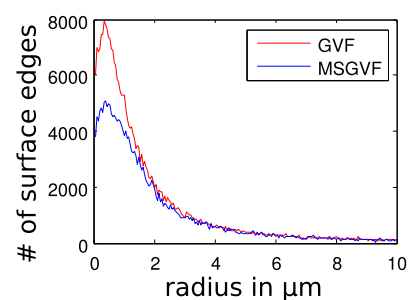

(b) Radii in the lower $20 \%$ of the surface.
Figure 4. Radii of the fitted spheres in the central and in the lower $20 \%$ of the segmentation mask.

that the surfaces are still more strongly curved in the lower regions than in the center. Though, compared to GVF, the new method works clearly better. For GVF, the ratio of the number of triangles forming an angle in a reasonable range (radii of $1-3 \mu \mathrm{m}$ ) and those forming too small angles (radii $<1 \mu \mathrm{m}$ ) is at 0.72 , for MSGVF it is at 0.92 . Having a closer look at the data (fig. 5, the red contour has been computed with GVF the green one with MSGVF), one can clearly see that in the central z-slices, the segmentation is very similar Only in the more difficult lower regions, the result found by MSGVF is smoother and shows less artifacts. For more examples, see [6].

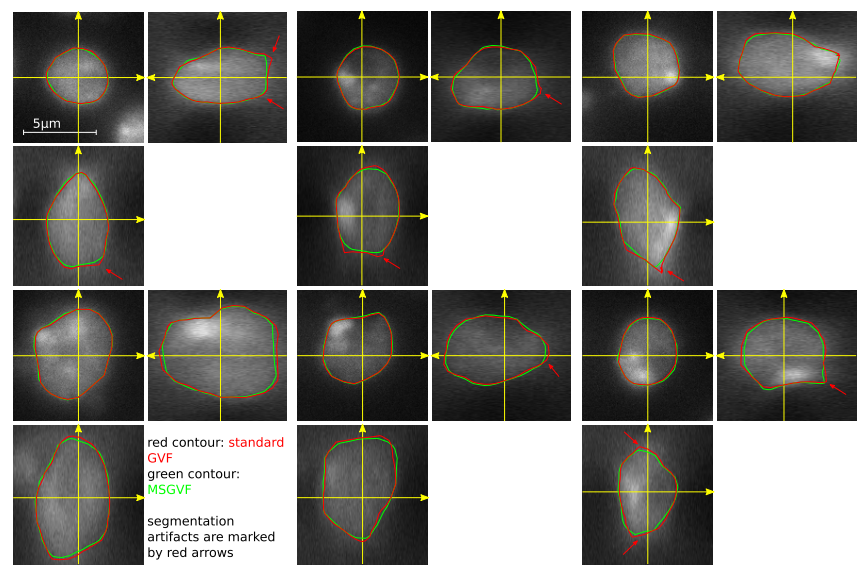

Figure 5. Results of the active surface segmentation for six nuclei.

\section{CONCLUSION}

We have presented a new method for the generation of external force fields for 2D and 3D active contours. The resulting vector fields are more robust against noise or smaller structures that should not influence the overall force field. Especially small but important gradients in gradient fields from blurred data are preserved. We could show that the segmentation of strongly blurred data from 3D widefield microscopy with an active surface model could be improved and the resulting surfaces show less PSF artifacts.

\section{ACKNOWLEDGMENT}

This study was supported by the Excellence Initiative of the German Federal and State Governments (EXC 294).

\section{REFERENCES}

[1] D. Cremers, Dynamical Statistical Shape Priors for Level Set Based Tracking, IEEE Trans. on PAMI, 28/8, 2006, 1262-1273.

[2] C. Xu and J. L. Prince, Snakes, shapes, and gradient vector flow, IEEE Trans. Imag. Proc.,7/3, 1998, 321-345.

[3] Y. Cheng, Mean Shift, Mode Seeking, and Clustering, IEEE Trans. on PAMI, 17/8, 1995,790-799.

[4] D. Comanciu and P. Meer, Mean Shift: A Robust Approach Toward Feature Space Analysis, IEEE Trans. on PAMI, 24/5, 2002, 603-619.

[5] M. Keuper, J. Padeken, P. Heun, H. Burkhardt and O. Ronneberger, A 3D Active Surface Model for the Accurate Segmentation of Drosophila Schneider Cell Nuclei and Nucleoli, Proc. of the ISVC, Springer LNCS, 2009, 865-874.

[6] M. Keuper, Mean Shifting Gradient Vector Flow, an Improved Force Field for Active Surfaces in Widefield Microscopy, Technical Report, IIF-LMB, University of Freiburg, 2010. 